HIV co-infection-are known to influence progression to fibrosis in the liver. Controversially, it has also been suggested that the use of cannabis (Cannabis sativa), a common recreational drug taken by at least $4 \%$ of the population in the US, has a role in hepatic fibrosis.

Ishida et al. examined data from a prospective cohort study of 204 patients with chronic $\mathrm{HCV}$ infection, $70 \%$ of whom had originally become infected via intravenous drug use. The degree of fibrosis detected in biopsy samples was compared with the reported frequency of cannabis use. Mild fibrosis was present in $27.5 \%$ of patients, moderate fibrosis was detected in $55.4 \%$ and the remaining $17.2 \%$ had severe fibrosis. Univariate and multivariate analyses showed that daily cannabis use was significantly associated with moderate to severe fibrosis. The presence of portal tracts in the liver was also an independent predictor of fibrosis, as was long-term moderate to heavy alcohol intake.

The authors conclude that HCV-infected patients who use cannabis on a daily basis are at a significantly increased risk of moderate or severe fibrosis. They predict that counseling $\mathrm{HCV}$-infected patients on the risks of cannabis use could be valuable.

Original article Ishida JH et al. (2008) Influence of cannabis use on severity of hepatitis C disease. Clin Gastroenterol Hepatol 6: 69-75

\section{Oral Aspergillus niger endoprotease therapy could be feasible}

A lifelong gluten-free diet effectively cures celiac disease, but compliance is difficult. Oral supplementation with nonhuman prolyl oligopeptidases (which cleave gluten proteins at proline residues and destroy their immunostimulatory properties) had long been proposed as a therapy for celiac disease, but most available enzymes did not function at gastric $\mathrm{pH}$, and were efficiently degraded by pepsin. Aspergillus niger endoprotease (AN-PEP) does not have these drawbacks; however, its proteolytic properties have not been tested in vivo.

Mitea and colleagues have now tested ANPEP in a computerized, mechanical system designed to simulate digestion in the human stomach and small intestine. They used data from healthy volunteers to model digestion in young adults after ingestion of a simple meal (white bread and water) and a complex fastfood meal (bread, hamburger, ketchup, French fries and soda), respectively. The researchers simulated digestion of each meal in the presence or absence of $200 \mathrm{mg}$ AN-PEP, and analyzed samples taken at various time points from the stomach, duodenal, jejunal and ileal compartments.

AN-PEP completely degraded the gluten present in both meals within $2 \mathrm{~h}$ (the average time for passage of food through the stomach), which greatly reduced the amount of T-cell-stimulatory epitopes derived from gliadins and glutenins that reached the duodenal compartment.

The authors note that gluten might be degraded even faster in vivo than in their system, which lacked proteolytic brush border enzymes. They call for clinical trials to determine whether oral AN-PEP supplementation can completely detoxify gluten.

Original article Mitea C et al. (2008) Efficient degradation of gluten by a prolyl endoprotease in a gastrointestinal model: implications for coeliac disease. Gut 57: 25-32

\section{SmartPill ${ }^{\circledR}$ versus scintigraphy for diagnosis of gastroparesis}

Delayed stomach emptying in patients with gastroparesis leads to nausea, vomiting, bloating, abdominal pain, discomfort and early satiety. An accurate diagnosis of gastroparesis can be achieved by gastric-emptying scintigraphy (GES), but this method requires exposure to radiation. A potential alternative to GES is the nondigestible SmartPill ${ }^{\circledR}$ capsule (SmartPill Corporation, Buffalo, NY), which records data on luminal $\mathrm{pH}$, temperature and pressure during gastrointestinal transit.

Kuo et al. assessed the ability of these two techniques to measure gastric emptying time (GET) and to distinguish healthy controls $(n=87)$ from patients with confirmed gastroparesis $(n=61)$. All participants fasted before ingesting a SmartPill ${ }^{\circledR}$ or a meal containing ${ }^{99 \mathrm{~m} T c-l a b e l e d}$ sulfur colloid. GES images were taken every $30 \mathrm{~min}$ for $6 \mathrm{~h}$.

GETs measured by the SmartPill ${ }^{\circledR}$ capsule correlated closely with those indicated by GES. The researchers noted a correlation of 0.73 between the percentage of the meal retained at $4 \mathrm{~h}$ ( $4 \mathrm{~h}$-GES, a standard cutoff point) and 